\title{
Prevalência de Desnutrição em Pacientes Adultos e Idosos em Fase de Pré-Tratamento de Leucemia
}

doi: https://doi.org/10.32635/2176-9745.RBC.2020v66n3.997

\author{
Malnutrition Prevalence in Adult and Older Adults Patients with Leukemia in Pretreatment Phase \\ Prevalencia de Desnutrición en Pacientes Adultos y Ancianos en la Fase Pretratamiento de la Leucemia
}

Thiago Huaytalla Silva'; Livia Costa de Oliveira²

RESUMO

Introduçáo: $\mathrm{O}$ estado nutricional em fase pré-tratamento pode estar relacionado a desfechos clínicos desfavoráveis em pacientes com câncer. Objetivo: Avaliar o estado nutricional de pacientes adultos e idosos com leucemia em fase de pré-tratamento oncológico. Método: Trata-se de um estudo transversal, retrospectivo, envolvendo pacientes com leucemia em fase pré-tratamento oncológico. Os critérios de inclusão foram $\geq 20$ anos de idade; ambos os sexos; e matrícula no Instituto Nacional de Câncer José Alencar Gomes da Silva (INCA). Os critérios de exclusão foram não ter diagnóstico confirmado de leucemia; registro de avaliaçáo do estado nutricional por meio da avaliação subjetiva global produzida pelo paciente; e não ter recebido tratamento oncológico prévio. Os testes qui-quadrado e $t$ de Student foram utilizados. Resultados: Foram avaliados 69 pacientes com leucemia em fase pré-tratamento oncológico, sendo em sua maioria homens $(52,2 \%)$ com menos de 60 anos de idade (52,2\%). A prevalência de desnutrição foi de $65,2 \%$, sendo mais expressiva nos idosos $(78,8 \%, p=0,023)$. A prevalência de risco nutricional apresentou a mesma frequência $(65,2 \%)$, no entanto, foi maior em pacientes com comorbidades $(80,8 \%, p=0,017)$ e história de tabagismo $(90,9 \%, p=0,056)$. Conclusáo: A maior parte dos pacientes com leucemia iniciou seu tratamento oncológico com o estado nutricional debilitado, o que se exacerbou entre aqueles com leucemia crônica, idosos, com comorbidades e história de tabagismo.

Palavras-chave: Leucemia; Estado Nutricional; Desnutrição; Prevalência; Tratamento Farmacológico.

\section{ABSTRACT}

Introduction: The nutritional status in the pretreatment phase may be related to unfavorable clinical outcomes in cancer patients. Objective: To assess the nutritional status of adult and older adults patients with leukemia in the pretreatment cancer phase. Method: Cross-sectional, retrospective study, involving patients with leukemia in the pretreatment cancer phase. The inclusion criteria were: $\geq 20$ years of age, both genders and enrollment at the National Cancer Institute José Alencar Gomes da Silva (INCA). The exclusion criteria were not having confirmed diagnosis of leukemia, registration of the Patient-Generated Subjective Global Assessment and not having submitted to previous cancer treatment. Chi-square and Student's t tests were used. Results: 69 patients were evaluated with leukemia in the pretreatment cancer phase, mostly men $(52.2 \%)$ under 60 years of age (52.2\%). The prevalence of malnutrition was $65.2 \%$, being more expressive in the older adults $(78.8 \%, \mathrm{p}=0.023)$. The prevalence of nutritional risk had the same frequency $(65.2 \%)$, however it was higher in patients with comorbidities $(80.8 \%, \mathrm{p}=0.017)$ and smoking history $(90.9 \%, \mathrm{p}=0.056)$. Conclusion: Most patients with leukemia started their cancer treatment with impaired nutritional status, which was exacerbated for those with chronic leukemia, older adults, with comorbidities and history of smoking. Key words: Leukemia; Nutritional Status; Malnutrition; Prevalence; Drug Therapy.
RESUMEN

Introducción: El estado nutricional en la fase de pretratamiento puede estar relacionado con resultados clínicos desfavorables en pacientes con cáncer. Objetivo: Evaluar el estado nutricional de pacientes adultos y ancianos con leucemia en fase de pretratamiento de cáncer. Método: Este es un estudio transversal, retrospectivo, que involucra a pacientes con leucemia en la fase de pretratamiento de cáncer. Los criterios de inclusión fueron $\geq 20$ años de edad; ambos os sexos; e inscripción en el Instituto Nacional del Cáncer José Alencar Gomes da Silva (INCA). Los criterios de exclusión fueron no tener un diagnóstico confirmado de leucemia; registro de la evaluación del estado nutricional a través de la Valoración Subjetiva Global-Generada por el Paciente; y no haber recibido tratamiento previo contra el cáncer. Se utilizaron las pruebas de Chi-cuadrado y $t$ de Student. Resultados: Se evaluaron 69 pacientes con leucemia en la fase de pretratamiento de cáncer, en su mayoría hombres $(52,2 \%)$ menores de 60 años $(52,2 \%)$. La prevalencia de desnutrición fue del $65,2 \%$, siendo más expresiva en los ancianos $(78,8 \%$, $\mathrm{p}=0,023)$. La prevalencia del riesgo nutricional tuvo la misma frecuencia $(65,2 \%)$, sin embargo, fue mayor en pacientes con comorbilidades $(80,8 \%$, $\mathrm{p}=0,017)$ y antecedentes de tabaquismo $(90,9 \%, \mathrm{p}=0,056)$. Conclusión: La mayoría de los pacientes con leucemia comenzaron su tratamiento con un estado nutricional deteriorado, que se exacerbó entre aquellos con leucemia crónica, ancianos, con comorbilidades y antecedentes de tabaquismo.

Palabras clave: Leucemia; Estado Nutricional; Desnutrición; Prevalencia; Quimioterapia.

'Especialista. Nutricionista. Hospital do Câncer IV (HC IV). Instituto Nacional do Câncer José Alencar Gomes da Silva (INCA). Rio de Janeiro (RJ), Brasil. Orcid iD: https://orcid.org/0000-0002-2415-9724

2Doutora. Nutricionista. Unidade de Cuidados Paliativos do INCA. Rio de Janeiro (RJ), Brasil. Orcid iD: https://orcid.org/0000-0002-5052-1846

Endereço para correspondência: Thiago Huaytalla Silva. Rua Visconde de Santa Isabel, 274 - Vila Isabel. Rio de Janeiro (RJ), Brasil. CEP $20560-120$. E-mail: thiagohuaytalla@gmail.com 


\section{INTRODUÇÃO}

A incidência e mortalidade por câncer são crescentes no mundo. Estimativas do Global Cancer Observatory mostram em 2018 uma incidência de leucemia de 249 mil casos para homens, ocupando a décima posição entre os tumores mais incidentes no mundo. Em relação às mulheres, são estimados 187 mil casos novos, sendo a décima segunda posiçăo ${ }^{1,2}$. No Brasil, estimam-se, para cada ano do triênio 2020-2022, 5.920 novos casos de leucemia em homens e 4.890 em mulheres ${ }^{3}$.

As leucemias são classificadas de acordo Organização Mundial da Saúde (OMS) em mieloides e linfoides, com base nos tipos de glóbulos brancos afetados. Também podem ser agudas ou crônicas, segundo o curso da doença. Desse modo, existem quatro principais leucemias: leucemia mieloide aguda (LMA); leucemia linfoblástica aguda (LLA); leucemia mieloide crônica (LMC); e leucemia linfocítica crônica (LLC) ${ }^{4,5}$. Entre essas, as mais prevalentes em adultos são as LMA, LMC e LLC 6 .

Sáo escassos os estudos que retratam o estado nutricional em pacientes adultos e idosos com leucemia, sendo a maioria das pesquisas realizada em crianças $^{7-9}$. Além disso, acredita-se que a desnutrição seja um problema sub-reconhecido no ambiente hospitalar ${ }^{10}$. A Sociedade Europeia de Nutrição Clínica e Metabolismo (ESPEN) e a Sociedade Americana de Nutrição Parenteral e Enteral (ASPEN) recomendam uma triagem precoce do estado nutricional em todos os pacientes hospitalizados ${ }^{11,12}$.

Entre as diversas ferramentas de avaliação do estado nutricional, a avaliação subjetiva global produzida pelo paciente (ASG-PPP) foi desenvolvida e validada para avaliação nutricional de pacientes oncológicos, sendo seu uso recomendado pela ESPEN e ASPEN ${ }^{11-13}$. Além disso, estudos recentes vêm demonstrando a relaçáo entre o estado nutricional debilitado, avaliado pela ASG-PPP, e os desfechos clínicos, como o prolongamento da internação hospitalar e a menor sobrevida em pacientes com câncer hematológico ${ }^{7,14}$.

Considerando a relevância da avaliação do estado nutricional e a escassez desse dado em pacientes com leucemia a partir da idade adulta e idosa, o objetivo deste trabalho foi avaliar o estado nutricional nesses grupos de pacientes, em fase de pré-tratamento oncológico.

\section{MÉTODO}

Trata-se de um estudo transversal, retrospectivo, com amostra de conveniência, conduzido por meio de análise de prontuários de pacientes com leucemia, matriculados na Clínica de Hematologia do Instituto Nacional de Câncer José Alencar Gomes da Silva (INCA), entre janeiro de 2009 e dezembro de 2013. O estudo foi aprovado pelo Comitê de Ética em Pesquisa da Instituição sob o número CAAE 14452919.0.0000.5274.

De acordo com os critérios de elegibilidade, os pacientes selecionados deveriam ter: $\geq 20$ anos de idade; diagnóstico confirmado de leucemia; matrícula no INCA no período de interesse; registro de avaliação do estado nutricional por meio da ASG-PPP; e não ter recebido tratamento oncológico prévio.

Os critérios de inclusão foram $\geq 20$ anos de idade; ambos os sexos; e matrícula no INCA no período de interesse. Já os critérios de exclusão foram não ter diagnóstico confirmado de leucemia; registro de avaliação do estado nutricional por meio da ASG-PPP; e não ter recebido tratamento oncológico prévio.

Pesquisadores treinados extraíram dados de interesse, referentes ao período de pré-tratamento, e os registraram em formulário específico, sendo eles: sexo (masculino $v$ s. feminino), idade ( $<60 v s . \geq 60$ anos), cor da pele (brancos vs. não brancos), escolaridade (até o ensino médio completo vs. superior), possuir vínculo empregatício ( $\operatorname{sim} v s$. não), renda individual ( $<1 v s . \geq 1$ salário-mínimo), presença de comorbidades - hipertensão arterial e diabetes mellitus ( $\operatorname{sim} v$ s. não), história de tabagismo (se fuma atualmente e/ou ex-fumante: sim vs. não), história de etilismo (se bebe atualmente e/ou se já bebeu álcool: sim vs. não) e ASG-PPP (classificação: A vs. B+C; escore global: <9 vs. $\geq 9$ pontos). Posteriormente, todos os dados da revisão dos prontuários físicos e eletrônicos foram digitados em planilha eletrônica em um software especializado.

A ASG-PPP foi aplicada por nutricionistas da instituição previamente treinados. A ferramenta é constituída por duas partes. A primeira envolve a avaliação de quatro diferentes domínios: i. Alteraçóes do peso corporal (escore $\leq 5$ pontos); ii. Ingestáo alimentar (escore $\leq 4$ pontos); iii. Presença de sintomas de impacto nutricional (escore $\leq 24$ pontos); e iv. Funcionalidade (escore $\leq 3$ pontos). A partir dessa parte da ferramenta, é gerado um escore global com a soma dos pontos de cada um dos quatro domínios, podendo variar de 0 a 36 pontos (quanto maior a pontuaçáo, maior o risco nutricional). Pacientes com um escore global $>9$ pontos foram considerados em risco nutricional ${ }^{13}$. A segunda parte abrange a história do paciente, com dados como diagnóstico, idade, demanda metabólica, uso de corticosteroides e exame físico, incluindo perda de gordura subcutânea, perda de massa muscular e edema ou ascite. Ao final da avaliação da ferramenta completa, cada paciente foi classificado como bem nutrido (A); com desnutrição moderada (B); ou severa (C).

A análise dos dados foi realizada no programa STATA - (Stata Data Analysis and Statistical Software; Stata Corp., 
College Station, Texas, USA), versão 13.0. As estatísticas descritivas foram expressas como porcentagem (\%) absolutas ou relativas e média e desvio-padrão (DP), conforme apropriado. O teste Kolmogorov-Smirnov foi utilizado para avaliar a distribuição das variáveis numéricas. Para avaliar as diferenças entre os grupos, de acordo com o tipo de leucemia (crônica e aguda) e o estado nutricional, foram utilizados o teste qui-quadrado para proporçóes para variáveis categóricas e o teste $t$ de Student para avaliar diferença entre médias do escore global da ASG-PPP e para cada tipo de leucemia. Os resultados foram considerados estatisticamente significativos quando $\mathrm{p}<0,05$, com intervalos com $95 \%$ de confiança.

\section{RESULTADOS}

Dos 313 indivíduos matriculados na Clínica de Hematologia do INCA, entre janeiro de 2009 e dezembro de 2013, após verificação no prontuário eletrônico, restaram 267 indivíduos. Destes, 210 prontuários físicos foram analisados e fizeram parte da amostra final 69 pacientes, sendo o principal motivo de exclusão não terem registro em prontuário da avaliação do estado nutricional por meio da ASG-PPP (45,0\%) (Figura 1).

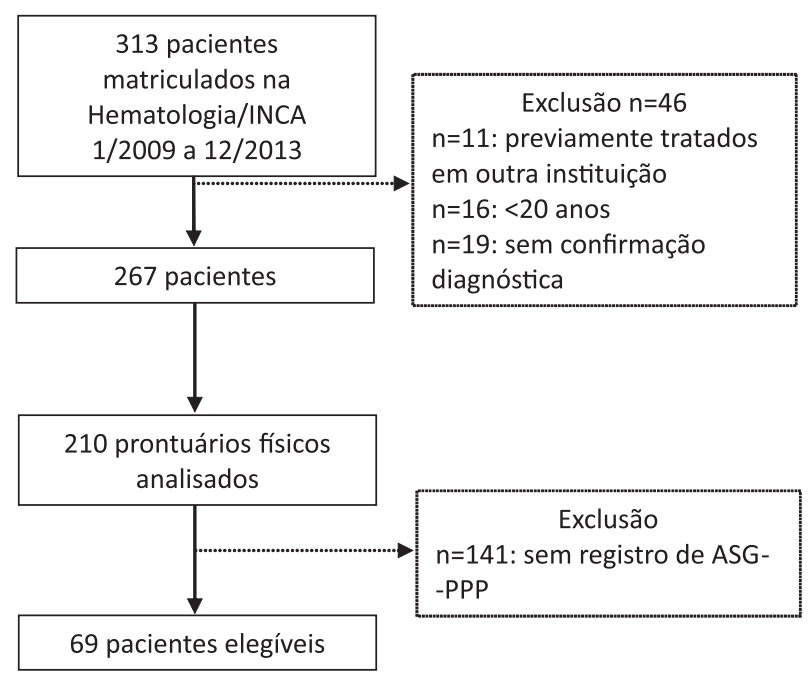

Figura 1. Fluxograma de seleção de pacientes

Legendas: INCA=Instituto Nacional de Câncer José Alencar Gomes da Silva. $\mathrm{n}=$ Número de observaçáo. ASG-PPP=Avaliação subjetiva global produzida pelo paciente.

A maioria dos pacientes com leucemia, em pré-tratamento oncológico, possuía menos de 60 anos de idade $(52,2 \%)$ e era do sexo masculino $(52,2 \%)$, com prevalência de desnutrição e risco nutricional na ordem de $65,2 \%$ (Tabela 1 ).

A amostra foi dividida em dois grupos: aqueles que tiveram diagnóstico de leucemia crônica $(n=33)$ e os que tiveram diagnóstico de leucemia aguda $(\mathrm{n}=36)$. No grupo com leucemia crônica, foi verificada maior prevalência de idosos $(\mathrm{p}=0,012)$ em risco nutricional $(\mathrm{p}=0,023)$ (Tabela 1$)$.

De acordo com a Figura 2, que demonstra o escore global da ASG-PPP segundo o tipo de leucemia, pacientes com leucemia crônica têm maior risco nutricional com pontuação mais elevado do que aqueles com leucemia aguda [13 (DP: 7) vs. 9 (DP: 5) pontos, $\mathrm{p}=0,034$ ).

A prevalência de desnutrição na amostra total foi maior em idosos $(78,8 \%$, p-valor=0,023). Por sua vez, a prevalência de risco nutricional foi maior em pacientes com comorbidades $(80,8 \%$, p-valor $=0,017)$ e história de tabagismo $(90,9 \%$, p-valor $=0,056)$ (Tabela 2$)$.

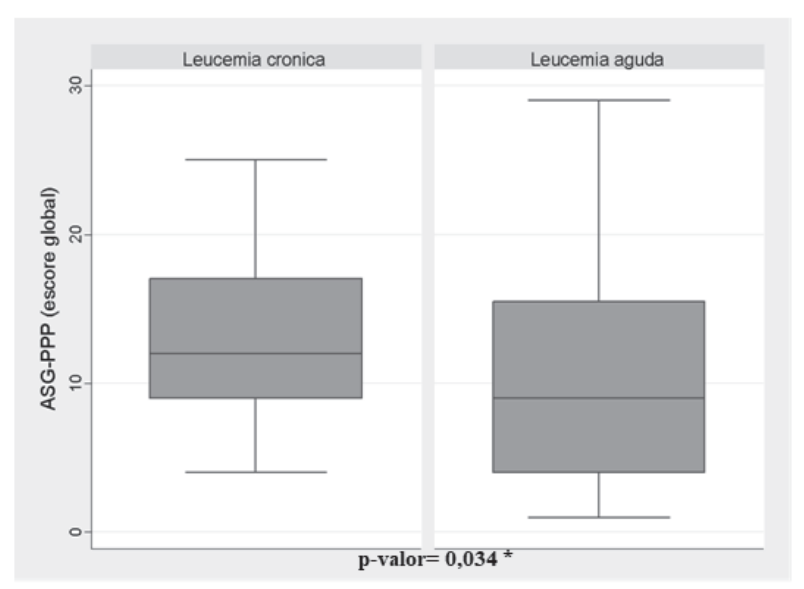

Figura 2. Escore global da avaliação subjetiva global produzida pelo paciente de acordo com o tipo de leucemia $(\mathrm{N}=69)$

Legendas: ASG-PPP=Avaliaçáo subjetiva global produzida pelo paciente. Escore global médio da ASG-PPP em pacientes com leucemia crônica=13 (desvio-padrăo: 7) pontos; aguda=9 (desvio-padráo: 5) pontos. ${ }^{*}$ p-valor refere-se ao teste $t$ de Student.

\section{DISCUSSÃO}

O presente estudo mostrou que a maior parte dos pacientes com leucemia já iniciou seu tratamento oncológico com estado nutricional debilitado, configurado pela presença de desnutriçáo e/ou risco nutricional em elevadas proporçôes. As prevalências dessas desordens foram mais expressivas entre aqueles com leucemia crônica, idosos, com comorbidades e história de tabagismo.

Sendo o estado nutricional um dos fatores potencialmente modificáveis e que está relacionado a desfechos clínicos desfavoráveis, seu monitoramento deve ser precoce, contínuo e individualizado em toda a trajetória das doenças. Trata-se de um elemento indispensável na fase de pré-tratamento oncológico para o diagnóstico precoce de distúrbios nutricionais, servindo como base para o planejamento de uma terapia nutricional especializada e individualizada ${ }^{13}$. 
Tabela 1. Prevalência de variáveis selecionadas de acordo com o tipo de leucemia $(N=69)$

\begin{tabular}{|c|c|c|c|c|}
\hline \multirow[b]{2}{*}{ Variáveis } & \multirow[b]{2}{*}{$\begin{array}{l}\text { Total } \\
\text { n (\%) }\end{array}$} & \multicolumn{2}{|c|}{ Leucemia } & \multirow[b]{2}{*}{ p-valor* } \\
\hline & & $\begin{array}{c}\text { Crônica }(n=33) \\
n(\%)\end{array}$ & $\begin{array}{c}\text { Aguda }(n=36) \\
n(\%)\end{array}$ & \\
\hline \multicolumn{5}{|l|}{ Idade (anos) } \\
\hline$<60$ & $36(52,2 \%)$ & $12(33,3 \%)$ & $24(66,7 \%)$ & \\
\hline$\geq 60$ & $33(47,8 \%)$ & $21(63,6 \%)$ & $12(36,4 \%)$ & 0,012 \\
\hline \multicolumn{5}{|l|}{ Sexo } \\
\hline Masculino & $36(52,2 \%)$ & $17(47,2 \%)$ & $19(52,8 \%)$ & \\
\hline Feminino & $33(47,8 \%)$ & $16(48,5 \%)$ & $17(51,5 \%)$ & 0,916 \\
\hline \multicolumn{5}{|l|}{ Cor da pele branca** } \\
\hline Sim & $38(55,9 \%)$ & $20(52,6 \%)$ & $18(47,4 \%)$ & \\
\hline Não & $30(44,1 \%)$ & $13(43,3 \%)$ & $17(56,7 \%)$ & 0,446 \\
\hline \multicolumn{5}{|l|}{ Escolaridade } \\
\hline$\leq$ ensino médio & $58(84,0 \%)$ & $28(48,3 \%)$ & $30(51,7 \%)$ & \\
\hline$\geq$ ensino superior & $11(16,0 \%)$ & $5(45,5 \%)$ & $6(54,5 \%)$ & 0,864 \\
\hline \multicolumn{5}{|l|}{ Vínculo de trabalho** } \\
\hline Sim & $26(41,9 \%)$ & $10(38,5 \%)$ & $16(61,5 \%)$ & \\
\hline Não & $36(58,1 \%)$ & $20(55,6 \%)$ & $16(44,4 \%)$ & 0,184 \\
\hline \multicolumn{5}{|l|}{ Renda individual** } \\
\hline$<1$ salário mínimo & $19(33,9 \%)$ & $12(63,2 \%)$ & $7(36,8 \%)$ & \\
\hline$\geq 1$ salário mínimo & $37(66,1 \%)$ & $21(56,8 \%)$ & $16(43,2 \%)$ & 0,645 \\
\hline \multicolumn{5}{|l|}{ Comorbidades** } \\
\hline Sim & $26(41,3 \%)$ & $13(50,0 \%)$ & $13(50,0 \%)$ & \\
\hline Não & $37(58,7 \%)$ & $20(54,0 \%)$ & $17(46,0 \%)$ & 0,751 \\
\hline \multicolumn{5}{|l|}{ Tabagismo** } \\
\hline Sim & $11(17,7 \%)$ & $6(54,5 \%)$ & $5(45,5 \%)$ & \\
\hline Não & $51(82,3 \%)$ & $24(47,1 \%)$ & $27(52,9 \%)$ & 0,652 \\
\hline \multicolumn{5}{|l|}{ Etilismo** } \\
\hline Sim & $19(31,1 \%)$ & $9(47,4 \%)$ & $10(52,6 \%)$ & \\
\hline Não & $42(68,9 \%)$ & $21(50,0 \%)$ & $21(50,0 \%)$ & 0,849 \\
\hline \multicolumn{5}{|l|}{ ASG-PPP (classificação) } \\
\hline A: sem desnutrição & $24(34,8 \%)$ & $9(37,5 \%)$ & $15(62,5 \%)$ & \\
\hline $\mathrm{B}+\mathrm{C}:$ desnutrição & $45(65,2 \%)$ & $24(53,3 \%)$ & $21(46,7 \%)$ & 0,210 \\
\hline \multicolumn{5}{|l|}{ ASG-PPP (escore global) } \\
\hline$<9$ : sem risco nutricional & $24(34,8 \%)$ & $7(29,2 \%)$ & $17(70,8 \%)$ & \\
\hline$\geq 9$ : risco nutricional & $45(65,2 \%)$ & $26(57,8 \%)$ & $19(42,2 \%)$ & 0,023 \\
\hline
\end{tabular}

Legendas: ASG-PPP=Avaliação subjetiva global produzida pelo paciente. *p-valor: Refere-se ao teste qui-quadrado para proporçôes. **Variáveis com dados faltantes, portanto, com frequência relativa.

A ASG-PPP destaca-se como um método apropriado para esse fim, tratando-se de uma ferramenta clínica, simples, pouco invasiva e de baixo custo, por meio da qual fatores adversos que influenciam o estado nutricional podem ser avaliados $^{15}$. Além disso, apesar da classificação subjetiva de desnutriçáo fornecida pela ferramenta (A, B ou C), seu escore global permite uma interpretação objetiva e um monitoramento mais preciso das variações individuais do estado nutricional, identificando pacientes que necessitam de intervenção nutricional urgente e auxiliando na prevenção ou redução dos sintomas durante todas as linhas de tratamento oncológico que possam ser deliberadas ${ }^{16}$.

Portanto, o conhecimento do estado nutricional do paciente com câncer é uma parte fundamental do 
Tabela 2. Variáveis selecionadas de acordo com o estado nutricional ( $N=69)$

\begin{tabular}{|c|c|c|c|c|c|c|}
\hline \multirow{2}{*}{ Variáveis } & \multicolumn{2}{|c|}{ Desnutrição* } & \multirow{2}{*}{ p-valor*** } & \multicolumn{2}{|c|}{ Risco Nutricional** } & \multirow{2}{*}{ p-valor*** } \\
\hline & Não & Sim & & Não & Sim & \\
\hline \multicolumn{7}{|l|}{ Idade (anos) } \\
\hline$<60$ & $17(47,2 \%)$ & $19(52,8 \%)$ & 0,023 & $16(44,4 \%)$ & $20(55,6 \%)$ & 0,078 \\
\hline$\geq 60$ & $7(21,2 \%)$ & $26(78,8 \%)$ & & $8(24,2 \%)$ & $25(75,8 \%)$ & \\
\hline \multicolumn{7}{|l|}{ Sexo } \\
\hline Masculino & $16(44,4 \%)$ & $20(55,6 \%)$ & 0,078 & $16(44,4 \%)$ & $20(55,6 \%)$ & 0,078 \\
\hline Feminino & $8(24,2 \%)$ & $25(75,8 \%)$ & & $8(24,2 \%)$ & $25(75,8 \%)$ & \\
\hline \multicolumn{7}{|l|}{ Cor da pele branca } \\
\hline Sim & $13(43,3 \%)$ & $17(56,7 \%)$ & 0,218 & $11(29,0 \%)$ & $27(71,0 \%)$ & 0,339 \\
\hline Não & $11(28,9 \%)$ & $27(71,1 \%)$ & & $12(40,0 \%)$ & $18(60,0 \%)$ & \\
\hline \multicolumn{7}{|l|}{ Escolaridade } \\
\hline$\leq$ ensino médio & $20(34,5 \%)$ & $38(65,5 \%)$ & 0,904 & $19(32,8 \%)$ & $39(67,2 \%)$ & 0,418 \\
\hline$\geq$ ensino superior & $4(36,4 \%)$ & 7 (63.6\%) & & $5(45,5 \%)$ & $6(54,5 \%)$ & \\
\hline \multicolumn{7}{|l|}{ Vínculo de trabalho } \\
\hline Sim & $12(46,2 \%)$ & $14(53,8 \%)$ & 0,210 & $12(46,2 \%)$ & $14(53,8 \%)$ & 0,210 \\
\hline Não & 11 (30.6\%) & $25(69,4 \%)$ & & $11(30,6 \%)$ & $25(69,4 \%)$ & \\
\hline \multicolumn{7}{|l|}{ Renda individual } \\
\hline <1 salário mínimo & $8(42,1 \%)$ & $11(57,9 \%)$ & 0,474 & $6(31,6 \%)$ & $13(68,4 \%)$ & 0,644 \\
\hline$\geq 1$ salário mínimo & $12(32,4 \%)$ & $25(67,6 \%)$ & & $14(37,8 \%)$ & $23(62,2 \%)$ & \\
\hline \multicolumn{7}{|l|}{ Comorbidades } \\
\hline Sim & $6(23,1 \%)$ & $20(76,9 \%)$ & 0,063 & $5(19,2 \%)$ & $21(80,8 \%)$ & 0,017 \\
\hline Não & $17(45,9 \%)$ & $20(54,1 \%)$ & & $18(48,6 \%)$ & $19(51,4 \%)$ & \\
\hline \multicolumn{7}{|l|}{ Tabagismo } \\
\hline Sim & $4(36,4 \%)$ & $7(63,6 \%)$ & 0,946 & $1(9,1 \%)$ & $10(90,9 \%)$ & 0,056 \\
\hline Não & $18(35,3 \%)$ & $33(64,7 \%)$ & & $20(39,2 \%)$ & $31(60,8 \%)$ & \\
\hline \multicolumn{7}{|l|}{ Etilismo } \\
\hline Sim & $8(42,1 \%)$ & $11(57,9 \%)$ & 0,396 & $6(31,6 \%)$ & $13(68,4 \%)$ & 0,753 \\
\hline Não & $13(30,9 \%)$ & $29(69,1 \%)$ & & $15(35,7 \%)$ & $27(64,3 \%)$ & \\
\hline
\end{tabular}

Legendas: *Diagnóstico de desnutriçáo definido por meio da classificação da avaliação subjetiva global produzida pelo paciente. ${ }^{* *}$ Risco nutricional definido por meio do escore global da avaliaçấo subjetiva global produzida pelo paciente. ${ }^{* *}$ p-valor: Refere-se ao teste qui-quadrado para proporçôes.

tratamento clínico. A prevalência de desnutrição em pacientes com câncer varia cerca de $20 \%$ a mais de $70 \%$ em estudos mundiais, com diferenças relacionadas à idade, ao tipo e ao estágio do câncer. Estima-se que as taxas de mortes de pacientes com câncer sejam de $10 \%$ a $20 \%$ atribuídas à desnutrição e não à malignidade da doença ${ }^{17,18}$. Além disso, a desnutrição está associada a desfechos desfavoráveis, como perda de massa muscular, comprometimento imunológico com aumento de infecçôes, estresse psicossocial, menor qualidade de vida, toxicidade do tratamento e maior tempo de internação hospitalar com consequente aumento do custo ${ }^{11}$.

Estudos demonstraram que a prevalência de desnutrição especificamente entre pacientes hematológicos na admissão hospitalar, utilizando a ASG-PPP, variou de $17,5 \%$ a $47,7 \%{ }^{14,19,20}$. Essa amplitude de valores pode ser justificada pelos diferentes tipos de câncer hematológicos compreendidos em tais amostras. Já em estudos que avaliaram somente pacientes com leucemia aguda, foram verificadas menores prevalências e com menores variaçōes, na ordem de $15 \%$ a $27,9 \% \%^{7,21}$.

Este estudo retratou mais altas prevalências de desnutriçáo e de risco nutricional $(65,2 \%)$ do que as reportadas anteriormente. Apesar da heterogeneidade clínica das leucemias crônicas, a amostra é composta unicamente por pacientes hospitalizados, sintomáticos e necessitados do início imediato do tratamento oncológico, o que, provavelmente, justifica a maior expressividade do 
déficit ou risco nutricional verificado. É reafirmada, dessa forma, a necessidade precoce de suporte nutricional em indivíduos com câncer ${ }^{11}$.

Além disso, verifica-se, entre os pacientes com leucemia crônica, uma maior proporção de idosos $(\mathrm{p}=0,012)$ em risco nutricional $(\mathrm{p}=0,023)$, com médias mais elevadas de escore global da ASG-PPP $(p=0,034)$. Tais leucemias são quase exclusivas de pessoas com mais de 40 anos, com destaque para LLC, que tem idade média no diagnóstico de mais de 70 anos. A maioria dos pacientes é diagnosticada em um estágio precoce e assintomático, iniciando a terapia anos depois, o que aumenta a idade média do primeiro tratamento oncológico ${ }^{6}$.

Em razão do envelhecimento da populaçáo e do aumento da expectativa de vida dos idosos, o número de pacientes idosos com leucemia crônica aumenta em paralelo. Estudos evidenciam que idosos com tumores malignos são mais propensos à desnutrição, à redução da funcionalidade, à diminuição das capacidades cognitivas e ao aumento da inflamação, acarretando perda de massa corporal magra, maior gasto energético e aumento da termogênese ${ }^{22-25}$.

Além disso, muitas doenças crônicas associadas ao idoso reduzem a ingestão e absorção de alimentos, o que afeta negativamente o estado nutricional ${ }^{26}$. Santo et al. ${ }^{27}$, em estudo brasileiro, relatam que $65,6 \%$ dos idosos com câncer apresentam dificuldades para se alimentar e os sintomas mais mencionados foram boca seca, constipaçáo intestinal, falta de apetite e saciedade precoce ${ }^{27}$. Outro estudo revelou ainda que mais da metade dos idosos com tumores hematológicos apresentou déficit no estado nutricional ${ }^{28}$.

Verifica-se, nestes achados, que a prevalência de desnutriçáo moderada (B) e grave (C) foi maior em idosos $(78,8 \%$, p-valor $=0,023)$. Corroborando estes resultados, um estudo desenvolvido no Brasil por Pinho et al. ${ }^{29}$, com 4.783 pacientes com diferentes tipos de câncer, mostrou que há maior risco de indivíduos mais velhos apresentarem desnutrição (OR: 1,83; IC: 1,59-2,11), também avaliada por meio da ASG-PPP ${ }^{29}$. Já um estudo com 68 pacientes com leucemia aguda mostrou que indivíduos com mais de 60 anos estão associados com desnutrição grave ${ }^{21}$.

$\mathrm{O}$ risco nutricional foi mais prevalente em pacientes com comorbidades $(80,8 \%$, p-valor $=0,017)$ e com história de tabagismo (90,9\%, p-valor=0,056). É sabido que pacientes com comorbidades preexistentes ao câncer ou com história de tabagismo apresentam condições fisiológicas e metabólicas específicas em que é mais frequente o diagnóstico de risco nutricional ${ }^{30,31}$.

A desnutrição repercuti em diversos desfechos desfavoráveis ${ }^{25,32}$, considerando ainda que o paciente aqui avaliado se encontra na fase de pré-tratamento e será submetido a terapêuticas oncológicas diversas que, potencialmente, exacerbam os déficits nutricionais, com consequente aumento do risco de desfechos desfavoráveis ${ }^{11}$. Desse modo, destaca-se a relevância do papel nutricionista na assistência nutricional precoce, junto a outros profissionais de saúde, para promover a melhora do estado nutricional desses pacientes antes mesmo do início do tratamento oncológico.

Os resultados do presente estudo podem auxiliar a traçar hipóteses para novas pesquisas sobre a importância da avaliação do estado nutricional em pacientes com leucemia. Algumas limitaçóes metodológicas precisam ser apontadas. O diagnóstico nutricional pela ASG-PPP pode ter com algum grau de comprometimento em virtude da possibilidade de viés de memória, uma vez que alguns pacientes podem apresentar dificuldade em responder com exatidáo ao domínio da perda de peso nos últimos seis meses e em especificar a ingestão alimentar durante o último mês. A amostra envolveu um número de pacientes relativamente pequeno, de uma única instituição e com diferentes tipos de leucemia. Portanto, é necessário o desenvolvimento de mais estudos, com amostras maiores, mais homogêneas e de múltiplos centros. Por causa do reduzido tamanho amostral, não foram realizadas análises estatísticas mais robustas. No entanto, como os dados sobre estado nutricional em pacientes com câncer hematológico são escassos, a força do trabalho está em explorar um campo pouco estudado e de relevância, utilizando uma ferramenta reconhecida internacionalmente com tradução e adaptação cultural.

\section{CONCLUSÃO}

Com esses achados, concluiu-se que a maior parte dos pacientes com diagnóstico de leucemia iniciou seu tratamento oncológico já com comprometimento nutricional (desnutriçáo e/ou risco nutricional), o que se exacerbou naqueles com leucemia crônica, idosos, com presença de comorbidades e história de tabagismo (se fuma atualmente e/ou ex-fumante).

Em adição, com base nos escores da ASG-PPP, a maioria dos pacientes necessitou de intervenção nutricional, destacando a importância do diagnóstico nutricional precoce e da atuação de uma equipe multidisciplinar, incluindo o nutricionista, para oferta de tratamento adequado, enfatizando a terapia nutricional.

\section{CONTRIBUIÇÕES}

Thiago Huaytalla Silva e Livia Costa de Oliveira contribuíram substancialmente na concepçáo e planejamento do estudo; na obtenção, análise e 
interpretação dos dados; assim como na redaçáo e revisão crítica; e aprovaram a versão final a ser publicada.

\section{AGRADECIMENTO}

À Raísa Santiago pelo auxílio na coleta de dados.

\section{DECLARAÇÃO DE CONFLITO DE INTERESSES}

Nada a declarar.

\section{FONTES DE FINANCIAMENTO}

Não há.

\section{REFERÊNCIAS}

1. Stewart BW, Wild CP, editors. World Cancer Report 2014. Lyon: IARC Press; 2014. Chapter 1.2, Bray F. Transitions in human development and the global cancer burden; p. 54-68.

2. Bray F, Ferlay J, Soerjomataram I, et al. Global cancer statistics 2018: GLOBOCAN estimates of incidence and mortality worldwide for 36 cancers in 185 countries. CA Cancer J Clin 2018;68(6):394-424. doi: https://doi. org/10.3322/caac. 21492

3. Instituto Nacional de Câncer José Alencar Gomes da Silva. Estimativa 2020: incidência de câncer no Brasil. Rio de Janeiro: INCA; 2019.

4. Pokharel M. Leukemia: a review article. IJARPB. 2012;2(3):397-407.

5. Swerdlow SH, Campo E, Harris NL, et al., editors. WHO classification of tumours of haematopoietic and lymphoid tissues. 4th ed. Lyon: IARC; 2017.

6. Howlader N, Noone AM, Krapcho M, et al., editors. SEER Cancer Statistics Review 1975-2014 [Internet]. Bethesda, MD: National Cancer Institute; 2017 Apr. [cited 2020 Apr 1]. Available from: https://seer.cancer. gov/csr/1975_2014/

7. Deluche E, Girault S, Jesus P, et al. Assessment of the nutritional status of adult patients with acute myeloid leukemia during induction chemotherapy. Nutrition. 2017;41:120-5. doi: https://doi.org/10.1016/j. nut.2017.04.011

8. Esfahani A, Ghoreishi Z, Abedi Miran M, et al. Nutritional assessment of patients with acute leukemia during induction chemotherapy: association with hospital outcomes. Leuk Lymphoma. 2014;55(8):1743-50. doi: https://doi.org/10.3109/10428194.2013.853766

9. Brinksma A, Huizinga G, Sulkers E, et al. Malnutrition in childhood cancer patients: a review on its prevalence and possible causes. Crit Rev Oncol Hematol. 2012;83(2):249-75. doi: https://doi.org/10.1016/j. critrevonc.2011.12.003
10. Vest MT, Papas MA, Shapero M, et al. Characteristics and outcomes of adult inpatients with malnutrition. JPEN J Parenter Enteral Nutr. 2018;42(6):1009-16. doi: https:// doi.org/10.1002/jpen.1042

11. Arends J, Bachmann P, Baracos V, et al. ESPEN Guidelines on nutrition in cancer patients. Clin Nutr. 2017;36(1):11-48. doi: https://doi.org/10.1016/j. clnu.2016.07.015

12. August DA, Huhmann MB. A.S.P.E.N. clinical guidelines: nutrition support therapy during adult anticancer treatment and in hematopoietic cell transplantation. JPEN J Parenter Enteral Nutr. 2009;33(5):472-500. doi: https://doi.org/10.1177/0148607109341804

13. Jager-Wittenaar H, Ottery FD. Assessing nutritional status in cancer: role of the patient-generated subjective global assessment. Curr Opin Clin Nutr Metab Care. 2017;20(5):322-29. doi: https://doi.org/10.1097/ MCO.0000000000000389

14. Kim HS, Lee JY, Lim SH, et al. Patient-generated subjective global assessment as a prognosis tool in patients with multiple myeloma. Nutrition. 2017;36:67-71. doi: https://doi.org/10.1016/j.nut.2016.06.009

15. McCallum PD, Polisena CG, editors. The clinical guide to oncology nutrition. Chicago, IL: American Dietetic Association; 2000. Chapter 2, Patient-generated subjective global assessment; p. 11-23.

16. Araújo dos Santos C, Rosa COB, Ribeiro AQ, et al. Patient-generated subjective global assessment and classic anthropometry: comparison between the methods in detection of malnutrition among elderly with cancer. Nutr Hosp. 2015;31(1):384-92. doi: https://doi. org/10.3305/nh.2015.31.1.7543

17. Wie GA, Cho YA, Kim SY, et al. Prevalence and risk factors of malnutrition among cancer patients according to tumor location and stage in the National Cancer Center in Korea. Nutrition. 2010;26(3):263-8. doi: https://doi.org/10.1016/j.nut.2009.04.013

18. Sesterhenn AM, Szalay A, Zimmermann AP, et al. [Significance of autopsy in patients with head and neck cancer]. Laryngorhinootologie. 2012;91(6):375-80. doi: https://doi.org/10.1055/s-0032-1306363 German.

19. Fiol-Martínez L, Calleja-Fernández A, de la Maza BP, et al. Comparison of two nutritional screening tools to detect nutritional risk in hematologic inpatients. Nutrition. 2017;34:97-100. doi: https://doi.org/10.1016/j. nut.2016.09.009

20. Calleja-Fernández A, de la Maza BP, Casariego AV, et al. Food intake and nutritional status influence outcomes in hospitalized hematology-oncology patients. Nutr Hosp. 2015;31(6):2598-605. doi: https://doi.org/10.3305/ nh.2015.31.6.8674

21. Li J, Wang C, Liu X, et al. Severe malnutrition evaluated by patient-generated subjective global assessment results in poor outcome among adult patients with 
acute leukemia: a retrospective cohort study. Medicine (Baltimore). 2018;97(3):e9663. doi: https://doi. org/10.1097/MD.0000000000009663

22. Yan XJ, Dozmorov I, Li W, et al. Identification of outcome-correlated cytokine clusters in chronic lymphocytic leukemia. Blood. 2011;118(19):5201-10. doi: https:/doi.org/10.1182/blood-2011-03-342436

23. Steemburgo T, Averbuch NC, Belin CHS, et al. Hand Grip Strength and nutritional status in hospitalized oncological patients. Rev Nutr. 2018;31(5):489-99. doi: https://doi.org/10.1590/1678-98652018000500006

24. Song C, Cao J, Zhang F, et al. Nutritional risk assessment by scored patient-generated subjective global assessment associated with demographic characteristics in 23,904 common malignant tumors patients. Nutr Cancer. 2019;71(1):50-60. doi: https://doi.org/10.1080/01635 581.2019 .1566478

25. Silva TH, Schilithz AOC, Peres WAF, et al. Neutrophillymphocyte ratio and nutritional status are clinically useful in predicting prognosis in colorectal cancer patients. Nutr Cancer. 2019:1-10. doi: https://doi.org/ 10.1080/01635581.2019.1679198

26. Vandewoude MFJ. [Nutritional assessment in oncogeriatrics]. Tijdschr Gerontol Geriatr. 2010;41(5):214-20. doi: https://doi.org/10.1007/ BF03096213 Dutch

27. Santos CA, Ribeiro AQ, Rosa COB, et al. Depressão, déficit cognitivo e fatores associados à desnutrição em idosos com câncer. Ciênc Saúde Coletiva. 2015;20(3):751-60. doi: https://doi.org/10.1590/141381232015203.06252014

28. Hamaker ME, Prins MC, Stauder R. The relevance of a geriatric assessment for elderly patients with a haematological malignancy - A systematic review. Leuk Res. 2014;38(3):275-83. doi: https://doi.org/10.1016/j. leukres.2013.12.018

29. Pinho NB, Martucci RB, Rodrigues VD, et al. Malnutrition associated with nutrition impact symptoms and localization of the disease: results of a multicentric research on oncological nutrition. Clin Nutr. 2019;38(3):1274-79. doi: https://doi.org/10.1016/j. clnu.2018.05.010

30. Ge T, Lin T, Yang J, et al. Nutritional status and related factors of patients with advanced lung cancer in northern China: a retrospective study. Cancer Manag Res. 2019;11:2225-31. doi: https://doi.org/10.2147/ CMAR.S193567

31. Fujiya K, Kawamura T, Omae K, et al. Impact of malnutrition after gastrectomy for gastric cancer on longterm survival. Ann Surg Oncol. 2018;25(4):974-83. doi: https://doi.org/10.1245/s10434-018-6342-8

32. Oliveira LC, Abreu GT, Lima LC, et al. Quality of life and its relation with nutritional status in patients with incurable cancer in palliative care. Support Care Cancer.
2020 Feb;28:4971-8. doi: https://doi.org/10.1007/ s00520-020-05339-7 